\title{
Phase Synchronization Enhance of Rank 3 Graphs
}

\author{
Tao Shaohua*, Qiu Yingyu and Zhang Zhili
}

School of Information Engineering, Xuchang University, Xuchang, Henan, 461000, P.R. China

\begin{abstract}
This work deals with the synchronization on Rank 3 graph. Rank 3 graphs have better symmetry properties. Reconstructing graph structure by utilized the orbit of symmetric properties of Rank 3 graph. Our main result in this paper is research the synchronization of Rank 3 graph by Kuramoto model, includes the lattice graph, triangular graph, and paley graph. The results show that the reduction Rank 3 graphs have better synchronization.
\end{abstract}

Keywords: Orbit, rank 3 graph, synchronization, symmetry graph.

\section{INTRODUCTION}

Graphs are the natural theoretical model of computational networks and have close relationship with real networks [13]. Network features can be formulated and discussed via different graphs, like regular graphs, complete graphs, and others. Essential feature of graph reflect to the network and match the real network properties on some extent. Therefore, the computational networks performed distributed algorithms and enable processors to perform independent computations and communication with others through links with network nodes [4]. Among different classes of graphs Rank 3 graph are of special interest [5], combining basic features of graph, such as trees and compete graphs. Rank 3 graphs include many graphs, this paper study a part of Rank 3 graphs are square lattice graph [4], triangular graph [1, 3, 4], paley graph [3]. Rank 3 graphs have rich symmetry. The more symmetry in graphs has, and the more automorphism of graph are $[6,7]$. The synchronization study on graph has aroused the researcher attention with different research fields [8-10]. How to optimize the synchronization on graph few paper study it. The goal of this paper is utilizing the orbit in partition of automorphism and reducing the Rank 3 graph scale to reach better synchronization of reduction graph. The results show that the reduction graphs of Rank 3 have better synchronization than the original Rank 3 graphs.

\section{SYMMETRY GRAPH}

\subsection{Symmetry Graph}

A graph or network is denoted by $H=(V, E)$, where $V$ is the set of vertices and $E$ is the set of edges. To any node $v \in V(G), \varepsilon(v)=v$, obviously, there has a isomorphism to identity map $\varepsilon: V(H) \rightarrow V(H)$ [11-13]. We illustrate the concept through (Fig. 1). There has a map $\alpha_{1}: V(H) \rightarrow V(H)$ defined by $\alpha_{1}(v)=v$, which is a isomorphism from $H$ to itself [11-13].
An autoorphisms of a graph $H$ is a isomorphism from $H$ to itself. We denote the set of automorphisms of graph $H$ under composition operation as $\operatorname{Aut}(\mathrm{H})$. Fig. (1) shows $\operatorname{Aut}(H)=\left\{\alpha_{1}, \alpha_{2}, \alpha_{3}, \alpha_{4}\right\}$. The automorphisms group also is a permutation group. The set of all of permutation group base on $n$ called symmetric group $S_{n}$. The network is considered as symmetry if its underlying graph contains not only an identity permutation.

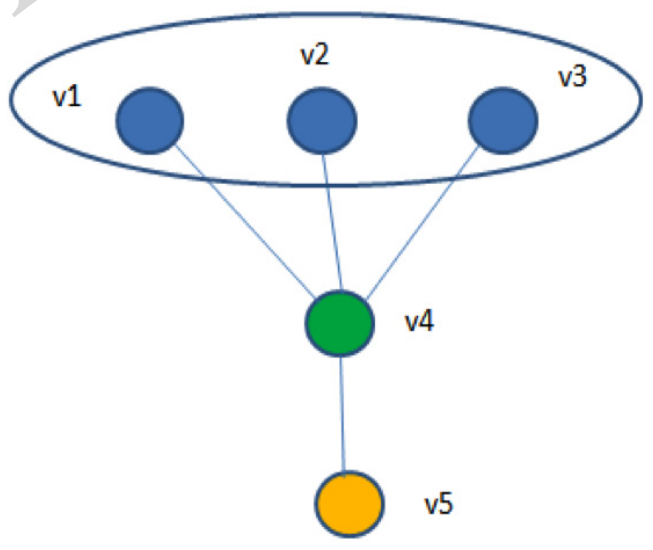

Fig. (1). Symmetry graph.

Next, we will introduce the concepts of automorphism partition and orbit. Given the automorphism group acting on vertex set $V$, we can get a partition $\pi=\left\{V_{0}, V_{1}, \ldots, V_{k}\right\}, x R y$ if and only if $\exists \alpha \in \operatorname{Aut}(H)$, s.t. $\alpha(x)=y$. Such partition is called automorphism partition, each cell of the partition is called an orbit of $\operatorname{Aut}(H)$, denote as $\operatorname{orbit}(H)$. An orbit is trivial if it only contains a single vertex, otherwise, the orbit is non-trivial. The vertex $u, v$ called similar if they are in the same orbit. Fig. (1) shows the automorphism partition $\pi=\left\{\left\{v_{1}, v_{2}, v_{3}\right\},\left\{v_{4}\right\},\left\{v_{5}\right\}\right\}$ and the orbits. Vertices in same orbit are marked with the same color in Fig. (1). The vertices in the same orbit have similarity [10]. Hence, we view them as a node, for example, $\left\{v_{1}, v_{2}, v_{3}\right\} \rightarrow v_{1},\left\{v_{4}\right\} \rightarrow v_{2},\left\{v_{5}\right\} \rightarrow v_{3}$, shows as Fig. (1) the dashed line. We can further reduce the 


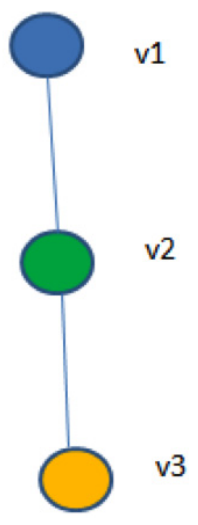

Fig. (2). Optimization graph.

network structure as Fig. (2). We call as optimization graph in this paper.

\subsection{Synchronization}

The synchronization of Kuramoto model [8, 14]:

$\frac{d \phi_{i}}{d t}=\omega_{i}-\frac{\delta}{N} \sum_{j} a_{i j} \sin \left(\phi_{i}-\phi_{j}\right)$

where $\phi_{i}$ and $\omega_{i}$ are the phase and the intrinsic frequency of vertex $i$, respectively. $\omega_{i}$ is chosen from the Gaussian distribution with unit variance. The summation runs over $j$, the nearest neighbors of vertex $i$. The coupling strength is used as $\delta$. The order parameter $M$ is defined as

$M=\left\{\left|\frac{1}{N} \sum_{j=1}^{N} e^{i \phi j}\right|\right\}$

where $\langle\ldots\rangle$ is the ensemble average over different configurations and $N$ is the total number of vertices. $M$ is $0(1)$ in the fully incoherent(coherent)phase.

The dynamics of synchronization on symmetry networks through a modified Kuramoto model, where orbit $[i]$ is the vertex $i$ in the same orbit.

$$
\frac{d \phi_{i}}{d t}=\omega_{i}-\frac{\delta}{\operatorname{Orbit}[i]} \sum_{j} a_{i j} \sin \left(\phi_{i}-\phi_{j}\right)
$$

\section{RANK 3 GRAPHS}

A rank 3 graph is of symmetry and is a graph whose automorphism group transitive on vertices, in other words, it is a orbit graph of a rank 3 permutation group of even order [7]. A rank 3 permutation group is synchronizing if the orbit is core [13]. In following, we analyze the synchronization of orbit of series of rank 3 graph, including of square lattice graph, Triangular graphs, Paley graphs and Line Graphs of Projective Plane.

\subsection{Square Lattice Graph}

A graph is said to be a lattice graph if it is a node-induced sub-graph of the infinite lattice, that is, its vertex set is a subset of $Z^{2}$ and two vertices are connected whenever they are at distance 1 [15]. The more usual definition of a lattice graph $L_{m, n}$ is the line graph of the complete bipartite graph $K_{m, n}$. This is the definition adopted for example by Brualdi and Ryser, although restricted to the case $m=n$. The lattice graph $L_{m, m}$ is also isomorphic to the latin square graph. The vertices of such a graph are defined as the $n^{2}$ elements of a latin square of order $n$, with two vertices being adjacent if they lie in the same row or column or contain the same symbol. The lattice graph $L_{2 n}$ is also the graph complement of the crown graph on $n$ vertices. A generalization of the square graph $s q_{n}$ is the graph obtained by taking the $n^{2}$ ordered pairs of the first $n$ positive integers as vertices and drawing an edge between all pairs having exactly one number in common. An example of the $s q_{3}$, shown as Fig. (3).

Here, the $s q_{3}$ as an example to calculate the orbits and analysis the synchronization. The orbits of $\operatorname{Aut}\left(s q_{3}\right)$ are $(\{18\},\{02\},\{35\},\{67\},\{4\})$.

Because in orbit nodes are similarity we optimized network topology by orbit. We revised the Kuramoto model as follow.

$$
\begin{gathered}
\frac{d \phi_{i}}{d t}=\omega_{i}-\frac{\delta}{N-\sum_{i=1}^{N} \operatorname{Orbit}[i]+\sum \text { orbit }} \sum_{j} a_{i j} \sin \left(\phi_{i}-\phi_{j}\right) \\
\sum_{i=1}^{N} \operatorname{Orbit}[i] \text { is the number of nodes in orbit and }
\end{gathered}
$$

$\sum$ Orbit $[i]$ is the number of orbit. We simulated the syn-

chronization of optimized network, original network and orbit by Eq. (3), as Fig. (4). Data1 represent the synchronization of orbit, data2 represent the synchronization of original network and data3 represent the synchronization of optimized network. We found that the synchronization of optimized network better than the original network.
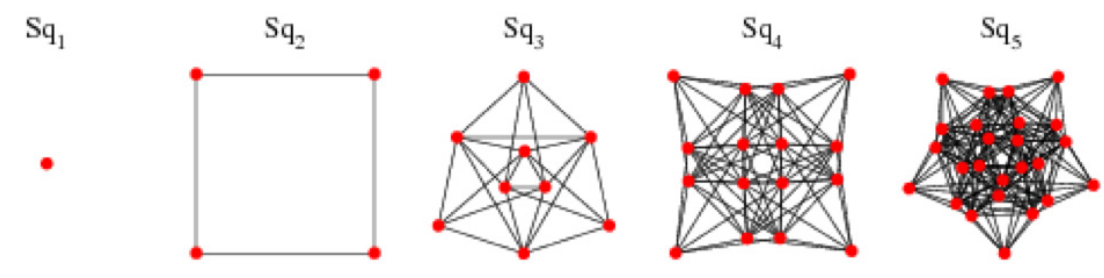

Fig. (3). Square lattice graphs. 


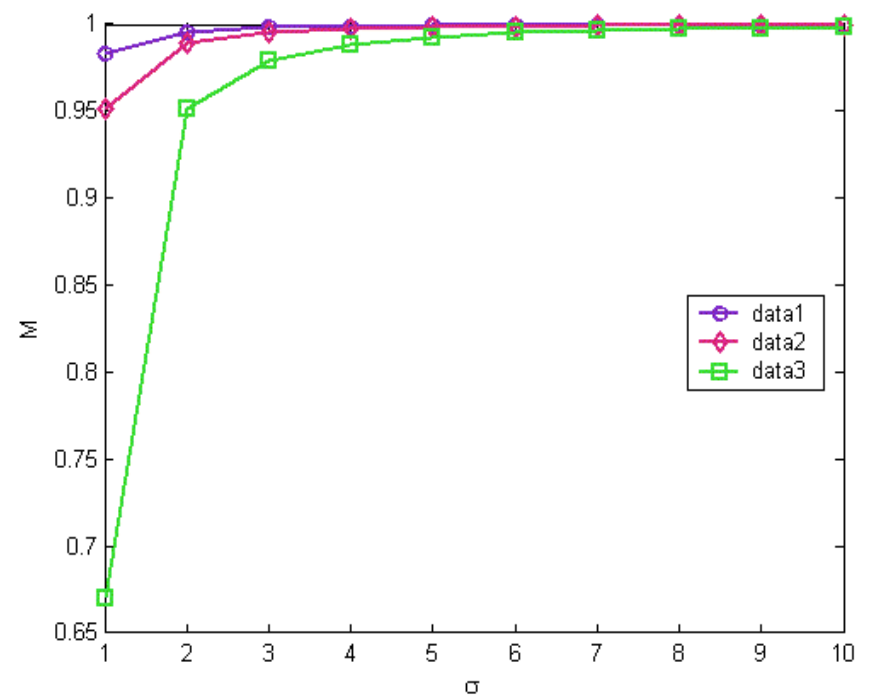

Fig. (4). Synchronization of optimization network, original network and orbit.

\subsection{Triangular Graphs}

Graph is triangular $T(n)$ if it is connected and each of its non-trivial cycle has a diagonal [15]. The vertices of $T(n)$ are the 2-element subsets of $\{1, \ldots, n\}$, two vertices are adjacent if the sets have non-empty intersection.

From the Fig. (5) we can known that each cycle in $T(n)$ contains a triangle, then connected graph $T(n)$ is triangular. Connected sub-graphs of triangular graphs also are triangular [15]. The symmetry group of $T(n)$ is $S_{n}$. Take $T_{4}$ as the example, the orbit of $\operatorname{Aut}\left(T_{4}\right)$ is $\left(\left\{\begin{array}{lll}0 & 3\end{array}\right\},\{124\}\right)$, each orbit has the 3 nodes. We also view node in orbit as one node, the graph only has the 2 nodes, is very easy to achieve to synchronization. According to the equation (1), (2) and (3), we simulated the synchronization of $T_{4}$, orbit of $\operatorname{Aut}\left(T_{4}\right)$ and optimization network, shown as Fig. (6). Data1 represent the synchronization of orbit, data2 represent the synchronization of original network and data3 represent the synchronization of optimized network.

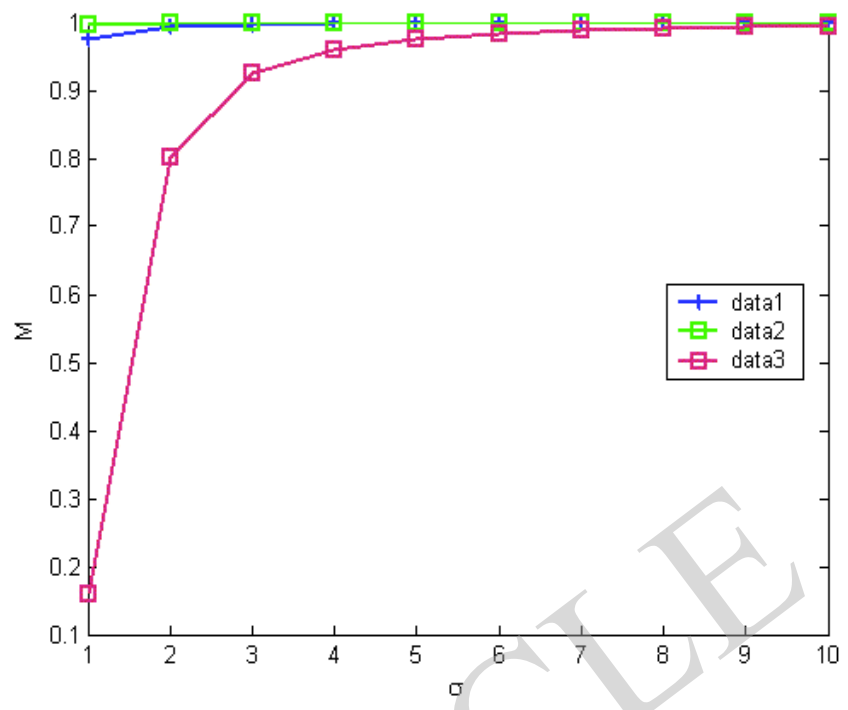

Fig. (6). Synchronization of optimization network, original network and orbit.

\subsection{Paley Graphs}

The paley graph $A(p)$ of order $q$ with $q$ a prime power is a graph on $q$ nodes with two nodes adjacent if their difference is a square in the finite field $G F(q)$ [19], shown as Fig. (7).

This is an undirected graph when $q$ is congruent 1 (mod 4), then $A(p)$ is symmetric [15]. The orbit of

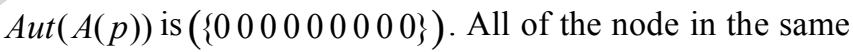
orbit, we needn't optimize the network structure. The network synchronization shows as

\subsection{Line Graphs of Projective Plane}

In mathematics a projective space is a set of elements similar to the set $P(V)$ of lines through the origin of a vector space $V$ [15]. The cases when $V=R^{2}$ or $V=R^{3}$ are the projective line and the projective plane, respectively. A line graph $L(G)$ also called an interchange graph or edge graph of a graph $G$ is obtained by associating a vertex with each
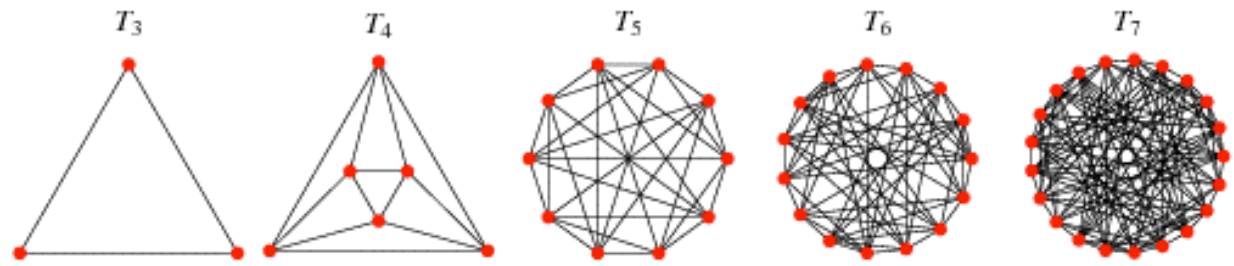

Fig. (5). triangular graphs.
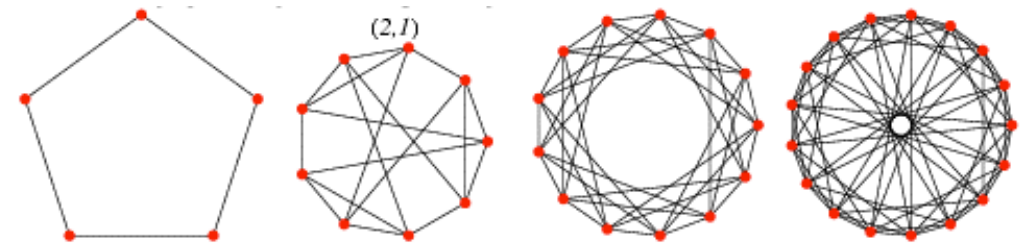

Fig. (7). Paley graph. 


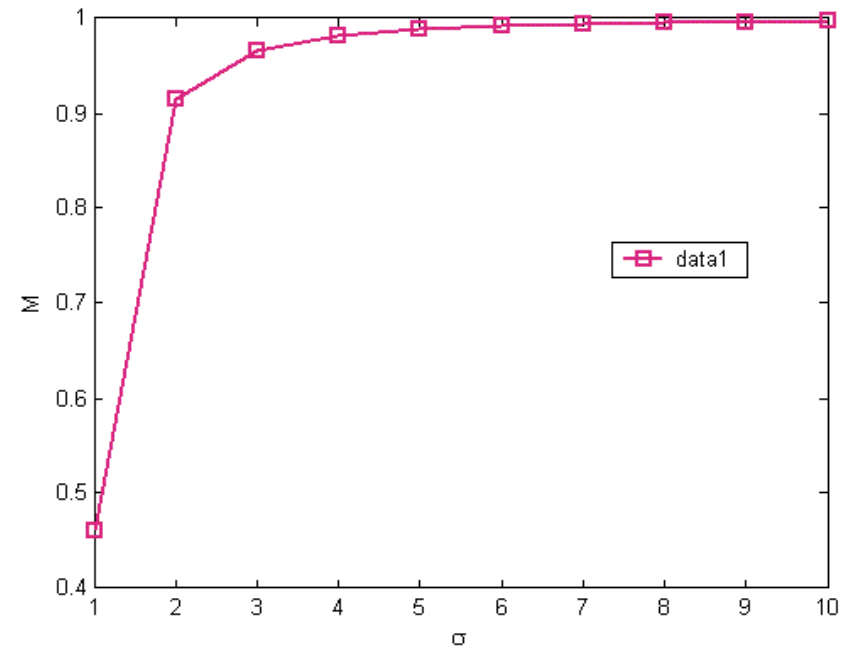

Fig. (8). Synchronization of orbit.

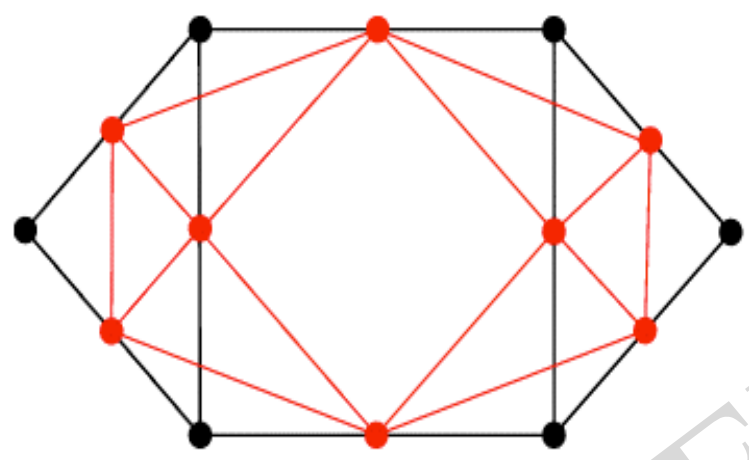

Fig. (9). Projective plane graph.

edge of the graph and connecting two vertices with an edge iff the corresponding edges of $G$ meet at one or both endpoints. An example graph (black) and its corresponding line graph (red) are illustrated as Fig. (9).

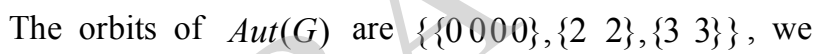
also simulated the synchronization of orbits, optimization network and the whole network, shown as Fig. (10). Data1 represent the synchronization of orbit of block node, data2 represent the synchronization of orbit of red node, data3 represent the synchronization of original network and data4 represent the synchronization of optimized network. The results show that the synchronization of optimized network better than original network synchronization.

\subsection{Polar Space}

In mathematics, in the field of combinatorics, a polar space of rank $n(n \geq 3)$, or projective index $n-1$, consists of a set $p$, conventionally the set of points, together with certain subsets of $p$, called subspaces, that satisfy these axioms [13]:

The polar space graph show as Fig. (11). In simulation, we take $N=9$ as the example, the orbits of $\operatorname{Aut}(G)$ are

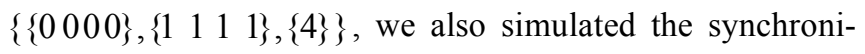
zation of orbits, optimization network and the whole network, shown as Fig. (12).

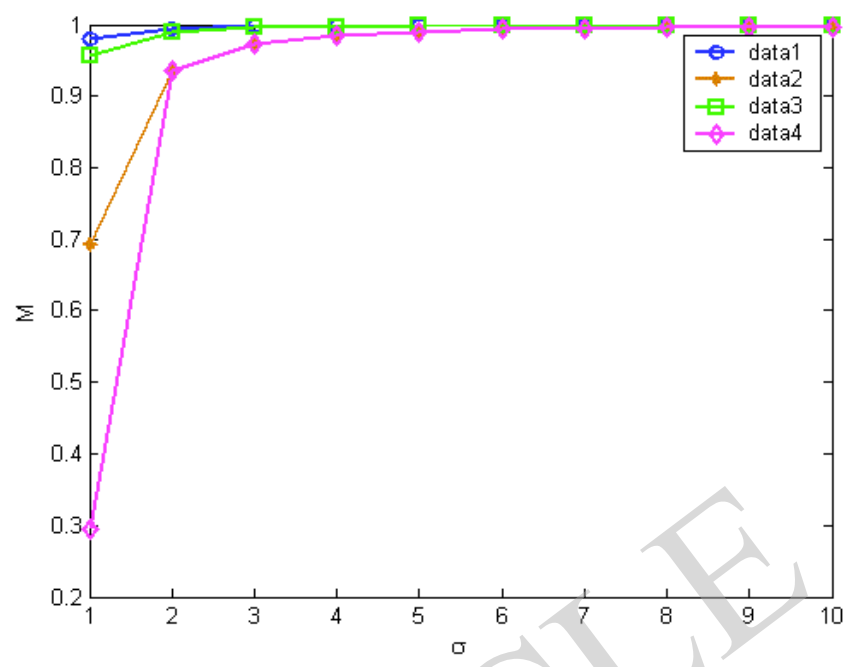

Fig. (10). Synchronization of optimization network, original network and orbit.

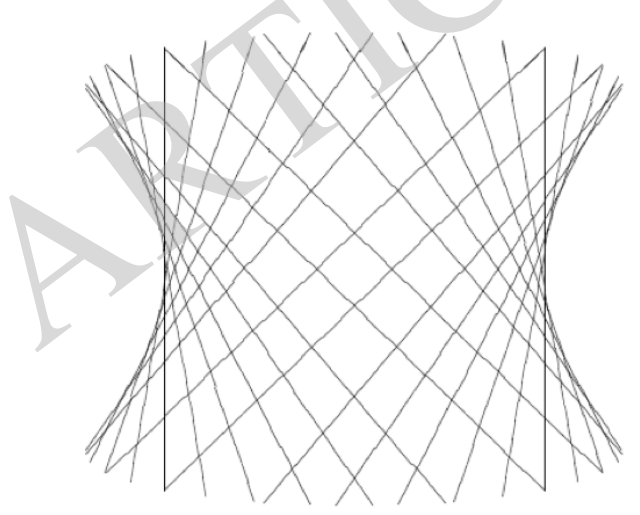

Fig. (11). Polar space graph.

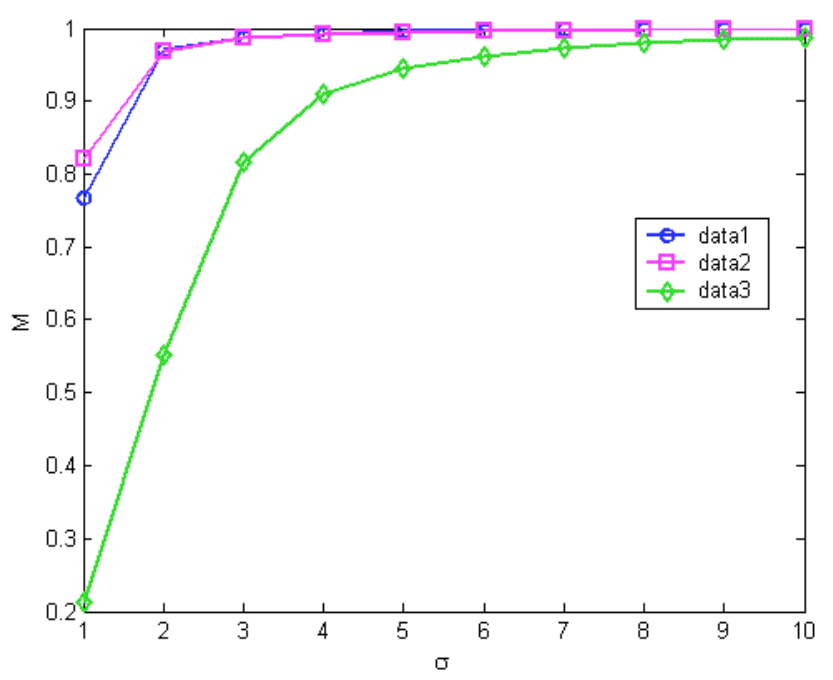

Fig. (12). Synchronization of polar space.

\section{CONCLUSION}

The paper studies the synchronization of symmetry graphs. We introduced the concept of symmetry of networks. If a network has more autoorphisms graphs and a network is more symmetry. On the other hands, nodes have similar 
properties in the orbit, we optimized the network structure by orbit, which is reducing the network size. This reduction network we called as optimization network. In this paper, first we analyze the synchronization of regular graph such as Rank 3 graphs. Some regular network, for example Square lattice graph, Triangular graphs, and Line Graphs of Projective Plane, these graphs have different orbits and enhance the synchronization is very easy. But Paley graphs, all nodes in the same orbit and we can't optimize the graph structure, but also reach the synchronization very well. The results show that symmetry structure can help to optimize the graphs topology and can enhance phase synchronization of graphs.

\section{CONFLICT OF INTEREST}

The authors confirm that this article content has no conflict of interest.

\section{ACKNOWLEDGEMENTS}

This work was supported in part by a grant from natural foundation of Henan (No.122102210544, No.12A510022 and No. 132300410349) and natural foundation (No. U1304403).

\section{REFERENCES}

[1] A. Mazurkiewica. "Local properties of triangular graphs", Proc. CS\&P'06, Wanditz, 2006, pp. 400-409.

[2] N. Dunford, and J. Schwartz, "Linear operators, Part I: general theory. Interscience Publisher: New York, 1958, pp. 217-274.

[3] http://mathworld.wolfram.com

[4] R. A. Bailey, "Latin squares with highly transitive automorphism groups", J. Aus. Math. Soc. Ser. A, vol. 33, pp. 18-22, 1982.

[5] M. Biliotti, and N. L. Johnson, "The non-solvable rank 3 affine planes", J. Combin. Theory Ser. A 93, vol. 45, pp.201-230, Jan. 2001.

[6] P. J. Cameron, Permutation Groups, London Mathematical Society Stuent Texts 45, Cambridge University Press, Cambridge, 1999, pp. 2000-2008.

[7] D. G. Higman, "Finite Permutation Groups of Rank 3", Math. Zeitschr. 86, Mich., USA, 1964, pp. 145-156.

[8] Y. Kuramoto, Chemical Oscillations, Wave and Turbulence, Springer-Verlag, Berlin 1984, pp. 1-158.

[9] X. Y. Zhang, and Y. Zou, "Explosive synchronization as a process of explosive percolation in dynamical phase space", Science Reports, vol. 4, pp. 5200, 2014

[10] Y. Zou, and T. Pereria, "Basin of attration determines hysteresis in explosive synchornization", PRL, vol. 112, pp. 114102, 2014.

[11] C. Godsil, G. Royle, Algebraic Graph Theory, Graduate Texts in Mathematics, Springer, 2001, pp. 443-520.

[12] N. Biggs, Algebraic Graph Theory, Cambridge University Press, London, 1974, pp. 9-14.

[13] P. J. Cameron, and P. A. Kazanidis, "Cores of symmetric graphs", J. Aus. Math. Soc., vol. 85, pp.145-154, 2008.

[14] A. Pikovsky, Synchronization, Cambridge Univ. Press, Cambridge, 2001, pp. 1-8.

[15] http://mathworld.wolfram.com/LatticeGraph.html

(C) Shaohua et al.; Licensee Bentham Open.

This is an open access article licensed under the terms of the Creative Commons Attribution Non-Commercial License (http://creativecommons.org/licenses/by-nc/3.0/) which permits unrestricted, non-commercial use, distribution and reproduction in any medium, provided the work is properly cited. 